\section{Proteus syndrome: a case report and a case study review in China}

\author{
Xi-Bao Zhang,' Chang-Xing Li,' \\ Yu-Qing He,' San-Quan Zhang,' \\ Yan-Xia Cai'
}

'Guangzhou Institute of Dermatology, Guangzhou, China;

2Dongguan Institute of Dermatology, Dongguan, China

\section{Abstract}

Proteus syndrome (PS) is a rare and sporadic disorder characterized by overgrowth of multiple tissues and a propensity to develop particular neoplasms. The clinical manifestations of PS include macrodactyly, vertebral abnormalities, asymmetric limb overgrowth and length discrepancy, hyperostosis, abnormal and asymmetric fat distribution, asymmetric muscle development, connective tissue nevi, and vascular malformations. We report a 16-year old female patient who manifested a number of these complications and review the Chinese literature about the diagnosis, natural history, and management of PS.

\section{Introduction}

The malformations in PS can involve skin, subcutaneous tissue, connective tissue (including bone), the central nervous system, and viscera. The main clinical manifestations of PS include hemihypertrophic macrodactyly, subcutaneous tumors, palmar and metatarsal cerebriform connective tissue nevi, lipomas, exogenesis bone mammilla, epidermal nevi, vascular malformations, lipohypoplasia, and dermalhypoplasia. ${ }^{1}$ In this article, we report a PS case that has a number of clinical manifestations, and review the literature on this unique disorder in China.

\section{Case report}

A 16-year old Chinese girl was born to nonconsanguineous parents. The pregnancy and delivery were uneventful. Her mother denied any drug use, radiation exposure, or infections during pregnancy. She presented to our institute at the age of 16 with facial dysmorphism and verrucous hyperplasia on the right side of her body. She was born with deafness of the right ear and dry, rough, light brown color patterns on the right trunk. As she grew, the patient developed more abnormalities, including right face, ear, tongue, and lip hyperplasia and overgrowth. The skin of the right trunk thickened and darkened, as the left side remained normal. Her parents, 3 sisters and brother are all normal, and there was no other similar disorder in her family.

\section{Physical examination}

The patient was noted to have normal intelligence. Her height and weight were $153 \mathrm{~cm}$ and $44 \mathrm{~kg}$, respectively. Her vital signs were within normal limits. The patient showed several anomalies. There was hemihypertrophy involving the entire right side of the head, including the skull, face, ear, palate, tongue, lip and neck. There was an epidermal nevus on the right side of the body, showing general cornification pachydermia. Hyperpigmented swirled and linear skin lesions were present on the right side of the neck and trunk, and verrucous epidermal nevi were seen on the right side of neck. She had malocclusion of the teeth, papillomarous hyperplasia of the tongue, hypertrophy of the right auricle, obstruction of the right auditory meatus, and a vascular malformation and lipomas on the right of the face. The distal right maniphalanx was enlarged and hammer-like. The right planta had an amber hyperplastic plaque that was moderately hard in texture and lacked pain sensation (Figures 1 and 2).

\section{Laboratory and auxiliary examination}

Computer tomography scan of the patient's head and face showed a large lipoma in the right face, but there were no abnormalities of the brain. Plain radiographs showed hyperplasia and hypertrophy of the jaw bone. There was a mild protrusion on the side of the thorax and the left tibia was mildly thickened. Electrocardiography was within normal limits. Ultrasounds for the other organs, such as liver, kidney, spleen, pancreas, uterus and both adnexa, were all within normal limits.

The histopathological changes in the epidermis were hyperplastic and affected chiefly the stratum corneum and stratum malpighii. Evaluation of the firm nodule adjacent to the right nasal ala revealed dense collagen consistent with a connective tissue nevus or linear verrucous epidermal nevus.

\section{Discussion}

\section{Etiology of Proteus syndrome}

The cause of PS is still unknown, but a genetic mutation that is viable only in a mosa-
Correspondence: Xi-Bao Zhang, Department of Dermatology, Guangzhou Institute of Dermatology, Guangzhou 510095, Guangdong Province, P R of China.

E-mail: lilichangxing@163.com

Key words: Proteus syndrome, clinical manifestation, diagnosis

Acknowledgments. The authors are deeply grateful to Dr. T Joseph and Dr. C Helena for their kind suggestions.

Conflict of Interest: the authors have no conflicts of interest to declare.

Received for publication: 2 November 2009 Revision received: 23 December 2009

Accepted for publication: 28 December 2009

This work is licensed under a Creative Commons Attribution 3.0 License (by-nc 3.0).

(C) Copyright X-B. Zhang et al., 2010

Licensee PAGEPress, Italy

Dermatology Reports 2010; 2:e3

doi:10.4081/dr.2010.e3

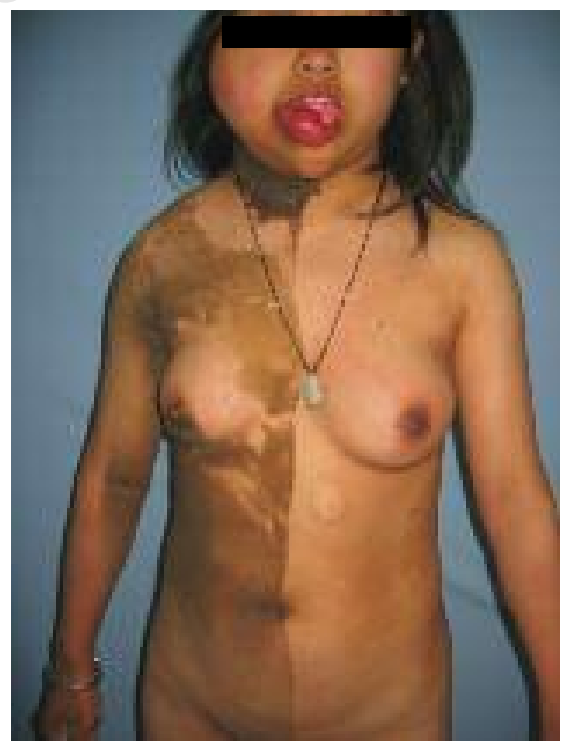

Figure 1. There was hemihypertrophy involving the entire right side of the head,including the skull, face, ear, palate, tongue, lip and neck.

ic state has been postulated. ${ }^{2}$ Such a mutation might affect local production or regulation of tissue growth factor receptors. This theory would explain the sporadic nature of the syndrome, its occurrence in various ethnic groups and both sexes, and its interindividual variability, as well as the mosaic pattern of lesion distribution in all who are affected.

In recent years, some authors have suggested that PS may be caused by germline mutations within the PTEN gene. ${ }^{3}$ The presence of a germline PTEN mutation in a subset of 


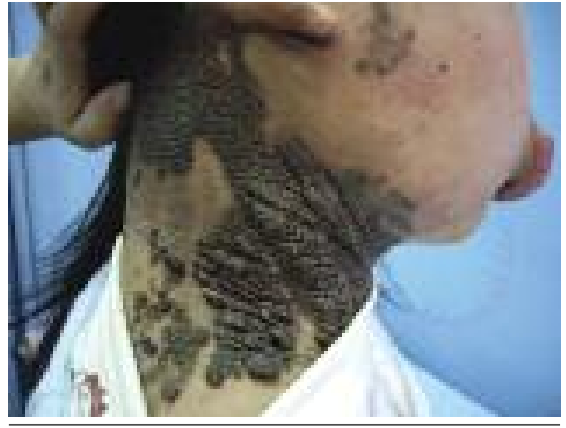

Figure 2. Verrucous epidermal nevi were seen on the right side of neck.

patients with PS has been confirmed by the identification of a de novo PTEN mutation in a patient with classical PS conforming to the criteria described by Biesecker et al. ${ }^{4}$

\section{Manifestations of Proteus syndrome}

PS is a rare and sporadic disorder that causes postnatal overgrowth of multiple tissues in a mosaic pattern. ${ }^{5}$ While patients with PS have a variable clinical appearance, they exhibit a defined constellation of skin abnormalities. Extracutaneous manifestations were divided into the following categories: skeletal overgrowth, visceral overgrowth, other overgrowth, tumors, cysts, vascular abnormalities, deformity, and hypoplasia/maldevelopment. ${ }^{6-8}$ Infants affected by the disorder usually appear normal or show only mild asymmetry at birth but progressively develop the characteristic features of the disease during childhood. ${ }^{9}$

Complications of PS include, among others, progressive skeletal deformities, invasive lipomas, benign and malignant tumors, and deep venous thrombosis with pulmonary embolism. Progressive skeletal abnormalities such as macrodactyly, scoliosis, asymmetric overgrowth, and limb length discrepancy are the most frequent and striking findings in patients with PS, followed by soft-tissue abnormalities such as fatty, muscular, and vascular malformations. Visceral anomalies such as splenomegaly, asymmetric megalencephaly, whitematter abnormalities, and nephromegaly as well as masses other than fatty, muscular, and vascular malformations are less common. ${ }^{10}$ The diagnosis of PS was based on published criteria. $^{4}$

\section{The current situation of investiga- tion on Proteus syndrome in China}

All 8 patients reported ${ }^{11-18}$ in China had at least 2 different skin abnormalities and had other abnormalities, including lipomas, telangiectatic nevi or other vascular lesions, plantar cerebriform connective tissue nevi, or linear lesions of an epidermal nevus. Remarkably, connective tissue nevi were also found on the

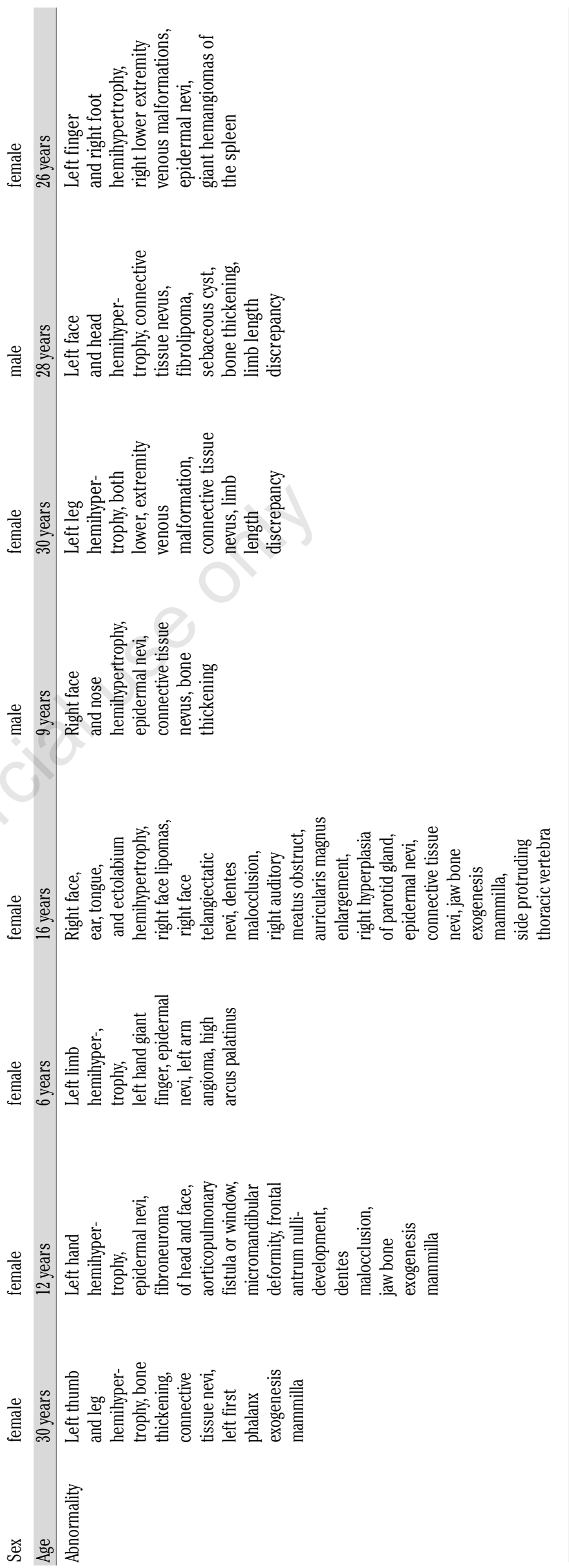


palms, forearms, trunk, and face. Epidermal nevi were examined histopathologically in all 8 patients (Table 1).

The reported cases were 6 females and 2 males; the youngest was six years old and the oldest was 30 years old. That only 8 cases have been reported in China suggests that many cases of PS may go unrecognized. China, as the most populated country on the globe at 1.2 billion people, should have more cases than have already been reported. The problem might be that both the clinical manifestations and diagnostic criteria are not familiar to dermatologists. Moreover, medical services are not as accessible to citizens because of China's status as a developing country. For these reasons, many cases may have been misdiagnosed or undiagnosed.

Although progress is being made in the clinical understanding of PS, much remains to be done. The existence of numerous case reports of patients who do not meet current clinical diagnostic criteria generates confusion about the natural history, the range of manifestations, and effective management techniques for what is now considered to be "true" PS.

\section{References}

1. Nguyen D, Turner JT, Olsen C, Biesecker LG, Darling TN. Cutaneous manifestations of Proteus syndrome: correlations with general clinical severity. Arch Dermatol. 2004;140:947-53.

2. Eng C, Thiele H, Zhou XP, Gorlin RJ, Hennekam RC, Winter RM. PTEN mutations and Proteus syndrome. Lancet. 2001;358:2079-80.

3. Cohen MM Jr, Turner JT, Biesecker LG. Proteus syndrome: misdiagnosis with PTEN mutations. Am J Med Gene. 2003;122A:323-4.

4. Biesecker LG, Happle R, Mulliken JB, Weksberg R, Graham JM Jr, Viljoen DL, et al. Proteus syndrome: diagnostic criteria, differential diagnosis, and patient evaluation. Am J Med Genet. 1999;84:389-95.

5. Happle R, Rogers M. Epidermal nevi. Adv Dermatol. 2002;18:175-201.

6. Biesecker LG, Peters KF, Darling TN, Choyke P, Hill S, Schimke N, et al. Clinical differentiation between Proteus syndrome and hemihyperplasia: description of a distinct form of hemihyperplasia. Am J Med Genet. 1998;79:311-8.

7. Happle R. Elattoproteus syndrome: delineation of an inverse form of Proteus syndrome. Am J Med Genet. 1999;84:25-8.

8. Fishman SJ, Mulliken JB. Vascular anomalies: a primer for pediatricians. Pediatr Clin North Am. 1998;45:1455-77.

9. Happle R, König A. Cutaneous mosaicism. Pediatric Dermatology. 2003:368-76.

10. Gilbert-Barness E, Cohen MM Jr, Opitz JM. Multiple meningiomas, craniofacial hyperostosis and retinal abnormalities in
Proteus syndrome. Am J Med Genet. 2000;93:234-40.

11. Ma Dong-Lai, Zuo Ya-Gang, Zheng He-Yi, Wang Bao-Xi. Proteus syndrome: a case report. J Clin Dermatol. 2005,34:205-7.

12. Chen Rengui, Jiang Yuanfang, Zhang Dingguo, Chen Ge. Proteus syndrome: A case report. J Clin Dermatol. 1995,4:242-3.

13. Yang Rui-Fang, Wang Ming-Yi, Wang JiZhou. Proteus syndrome: a case report. Chinese Journal of Birth Health \& Heredity. 1998,6:93-4.

14. He Yu-Qing, Zhang San-Quan, Cai YanXia, Zhang Xi-bao. Proteus syndrome: one case report. Chin J Dermatol. 2007,40:76970 .

15. Wang Qing-Fang, Li Chen-Jin, Sun Lin, Yu Feng-Zhang, Pan Shao-Chuan. Proteus syndroome: a case report. Chin J Pediatr Surg. 2007,28:669-70.

16. Luo Su-Ju, Feng Yi-Guo, Wang Jun-Ming, Zhen Yan, Peng Zhen-Hui, Liu Chao. Chin J Med Genet. 2006,23:366-7.

17. Lu Jiang-yang, Wang Xiao-hong, Liu Qian, Yang Yi, Li An-ran. Proteus syndrome: a clinicopathological observation. 2007;14: 339-43.

18. Wang Zhao-yue, SU Yan-hua, Yang Haiyan, Yu Zi-qiang, Cao Li-juan, Zhao Xiaojuan, et al. Proteus syndrome with a giant hemangiomas in the spleen associated with chronic DIC - two case report and literature review. Chin J Hematol. 2007; 28: 152-5. 